Parasitology (2006), 133: 673-684 Cambridge University Press

doi:10.1017/S003118200600117X

\title{
Parasite genetic diversity does not influence TNF-mediated effects on the virulence of primary rodent malaria infections
}

\author{
G. H. Long*, B. H. K. Chan, J. E. Allen, A. F. Read and A. L. Graham.
}

Institutes of Evolution, Immunology and Infection Research, School of Biological Sciences, University of Edinburgh, King's Buildings, West Mains Road, Edinburgh, EH9 3JT, Scotland.

Running Title: TNF- $\alpha$ and rodent malaria genotype

*Corresponding author:

Gráinne H. Long

Institutes of Evolution, Immunology and Infection Research,

School of Biological Sciences, University of Edinburgh

King's Buildings, West Mains Road

Edinburgh, EH9 3JT, Scotland.

$\mathrm{Ph}:$ (44) 131-650-5484

Fax: (44) 131-650-6564

E-mail: grainne.long@ed.ac.uk 
Parasitology (2006), 133: 673-684 Cambridge University Press

doi:10.1017/S003118200600117X

\section{SUMMARY}

The pro-inflammatory cytokine tumour necrosis factor alpha (TNF- $\alpha$ ) is associated with malarial virulence (disease severity) in both rodents and humans. We are interested in whether parasite genetic diversity influences TNF-mediated effects on malaria virulence. Here, primary infections with genetically distinct Plasmodium chabaudi chabaudi (P.c.c.) clones varied in the virulence and cytokine responses induced in female C57BL/6J mice. Even when parasitaemia was controlled for, a greater day 7 TNF- $\alpha$ response was induced by infection with more virulent P.c.c. clones. Since many functions of TNF- $\alpha$ are exerted through TNF receptor 1 (TNFR1), a TNFR-1 fusion protein (TNFR-Ig) was used to investigate whether TNFR1 blockade eliminated clone virulence differences. We found that TNFR-1 blockade ameliorated the weight loss, but not the anemia induced by malaria infection, regardless of P.c.c. clone. We show distinct P.c.c. infections induced significantly different plasma interferon gamma (IFN- $\gamma$ ), interleukin 6 (IL-6) and interleukin 10 (IL-10) levels. Our results demonstrate that regardless of P.c.c. genotype, blocking TNFR1 signalling protected against weight loss, but had negligible effects on both anemia and asexual parasite kinetics. Thus, during P.c.c. infection, TNF- $\alpha$ is a key mediator of weight loss, independent of parasite load and across parasite genotypes.

Key words: malaria, Plasmodium chabaudi, tumour necrosis factor- $\alpha$ (TNF- $\alpha$ ), parasite genotype, virulence, immunopathology, disease severity, cachexia 
Parasitology (2006), 133: 673-684 Cambridge University Press

doi:10.1017/S003118200600117X

\section{INTRODUCTION}

Malaria represents an enormous global health problem, causing immense morbidity and mortality (defined here as virulence) worldwide (Snow et al. 2005). Malaria infection causes a wide spectrum of virulence, resulting in an estimated 2 million deaths each year (Alles H K 1998). We are interested in understanding the causes of this variation in virulence. Both field and rodent studies have shown that while some virulence is directly linked to parasite load (Langhorne et al. 1998; Mackinnon \& Read 2004), some is immunopathological, arising as a result of the host's immune response to the parasite (Dodoo et al. 2002; Torre et al. 2002). The 'cytokine theory' of malaria, for example, hypothesizes that cytokines contribute to disease (Clark et al. 2004), and the potent pro-inflammatory cytokine tumor necrosis factor alpha (TNF- $\alpha$ ) has been shown to have dual roles in malaria infection: inhibiting in vivo parasite growth and controlling infection on the one hand (Jacobs et al. 1996; Langhorne et al. 1989; Li \& Langhorne 2000; Sam et al. 1999; Stevenson et al. 1995), while causing immune-mediated disease on the other (Clark \& Chaudhri 1988a; Clark \& Chaudhri 1988b; Grau et al. 1987; Kern et al. 1992).

We are particularly interested in whether host immune response contributes to the different virulence outcomes induced by genetically distinct P.c.c. infections. Studies using Plasmodium chabaudi chabaudi (P.c.c.), the immunologically best characterized rodent model of malaria, have focused largely on a single clone: AS (Jacobs et al. 1996; Langhorne et al. 1989; Li \& Langhorne 2000; Sam et al. 1999; Stevenson et al. 1995). However, primary infections by other P.c.c. clones have been shown to induce a spectrum of virulence phenotypes in a given inbred mouse strain supporting a role for 
Parasitology (2006), 133: 673-684 Cambridge University Press

doi:10.1017/S003118200600117X

parasite strain in virulence determination (Ferguson et al. 2003; Mackinnon \& Read 1999a; Mackinnon \& Read 1999b). Strain-specific variation in malaria virulence during human P. falciparum infections has also been reported (Chotivanich et al. 2000; Gravenor et al. 1995). Because high serum TNF- $\alpha$ concentrations are associated with severe infection (Grau et al. 1989; Molyneux et al. 1993; Molyneux et al. 1991) and malaria parasites directly stimulate the production of TNF- $\alpha$ from macrophages (Bate $\&$ Kwiatkowski 1994; Bate et al. 1992; Bate et al. 1988; Bate et al. 1989), we hypothesized that parasite genetic differences in induction of systemic TNF- $\alpha$ may contribute to clone differences in virulence. For example, distinct P.c.c. clones may achieve high virulence by (i) growing to high parasite densities in the host, (ii) inducing a large TNF- $\alpha$ response, or (iii) through a combination of these factors.

Ignoring the role parasite genotype plays in determining immune-mediated virulence may lead to conclusions which are parasite strain- or clone-specific. In the field of Leishmania, the role mouse strain played in disease outcome was the initial focus and led to conflicting data until it was recognized that parasite genotype could help to explain reported differences in disease outcome (Kebaier et al. 2001; Ritter et al. 2004). For example, infection with a range of $L$. major strains in $\mathrm{BALB} / \mathrm{c}$ mice resulted in distinct immune responses and a spectrum of virulence, from asymptomatic to visceral leishmaniasis (Kebaier et al. 2001). In addition, the anaemia induced during rodent Trypanosoma infections (Naessens et al. 2005) and the outcome of toxoplasmosis in rodent models (Mordue et al. 2001) have been shown to depend on the immune response induced by the particular host-parasite combination. For malaria, numerous studies have shown that mouse strains vary in their ability to control and clear primary infections (Li 
et al. 2001). However, immunological analysis of the role of parasite strain in determining the outcome of malaria has largely been overlooked, with the majority of rodent studies focusing on a single parasite strain or clone from a given Plasmodium species.

In the present study, we took two approaches to understand the role TNF- $\alpha$ plays in response to infection with distinct malaria genotypes. First, we investigated whether the level of virulence induced by the genetically distinct P.c.c clones correlated with a specific host cytokine response. We found that more virulent clones induced a greater plasma TNF- $\alpha$ response. To directly test whether the induction of a large TNF- $\alpha$ response is the reason some clones are more virulent, we then blocked TNF- $\alpha$ signalling by administering a soluble form of the TNF Receptor 1 (TNFR-Ig) and followed in vivo virulence, parasite dynamics and cytokine responses during distinct P.c.c. infections. We found that blocking TNFR-1 signalling protected against weight loss, but the level of protection did not differ among clones.

\section{MATERIALS AND METHODS}

\section{Mice and parasites}

Plasmodium chabaudi chabaudi (P.c.c.) clones were originally derived from thicket rat isolates and cryopreserved (Walliker et al. 1971). Clones are stored as frozen stabilities, with subscript codes denoting their exact clonal history. In both experiments we used the following clones; $\mathrm{CW}_{640}, \mathrm{AS}_{11915}, \mathrm{BC}_{200}$ and $\mathrm{AJ}_{4762}$ (herein referred to as $\mathrm{CW}, \mathrm{AS}, \mathrm{BC}$ and AJ). These four genetically distinct P.c.c. clones were selected based on the range of virulence they induce in C57BL/6 female mice; clones BC \& AJ are virulent and clones 
AS \& CW substantially less virulent in terms of weight and red blood cell loss (Mackinnon \& Read 1999a). Clone differences in virulence are maintained across mouse strain (de Roode et al. 2005; Mackinnon et al. 2002). In both experiment one and two, hosts were 6-8 week old female inbred C57BL/6 mice (Harlan, U.K. or bred at animal facilities of the University of Edinburgh, respectively). Mice were housed in filter-top cages, maintained in a $12 \mathrm{hr}: 12 \mathrm{hr}$ light-dark cycle, fed 41B maintenance diet (Harlan, U.K.) and their drinking water was supplemented with $0.05 \%$ para-amino benzoic acid (Jacobs 1964).

\section{Experimental design, parasite inoculation and in vivo antibody treatment}

Infections were initiated with an i.p. injection of $0.1 \mathrm{ml}$ inoculum containing $10^{6}$ red blood cells (RBCs) parasitized by one of the above four malaria clones. The inoculum was prepared from parasite donor mice through the dilution of infected blood in calf serum solution $\left(50 \%\right.$ Ringer's solution [27mM KCL, $\left.27 \mathrm{mM} \mathrm{CaCl}_{2}, 0.15 \mathrm{M} \mathrm{NaCl}\right], 50 \%$ heatinactivated calf-serum, 20 units heparin/ml mouse blood). Control mice received a $0.1 \mathrm{ml}$ inoculum of $10^{6}$ naïve $\mathrm{RBCs}$ and were subject to the same treatment regimes as experimental mice.

In experiment one, we investigated whether clone differences in malaria virulence could be explained by differences in plasma levels of TNF- $\alpha$, interleukin [IL]-6, IL$12 \mathrm{p} 70, \mathrm{IL}-10$ or interferon [IFN]- $\gamma$ in unmanipulated hosts on days 2,5 or 7 post infection (p.i.). Three mice per parasite clone were sacrificed at each time-point and plasma was collected for cytokine measurement. These time-points have previously been associated 
Parasitology (2006), 133: 673-684 Cambridge University Press

doi:10.1017/S003118200600117X

with protective or pathological cytokine responses in P.c.c. AS infections (Li et al. 1999; Li et al. 2003; Sam \& Stevenson 1999; Su \& Stevenson 2002).

In experiment two, the effect of blocking TNFR1 signalling on infection dynamics was investigated via the administration of a soluble TNF Receptor. Soluble TNF Receptor 1 (herein referred to as TNFR-Ig) has been shown to bind and efficiently inhibit mouse TNF- $\alpha$ activity in vivo (Ashkenazi et al. 1991) and was kindly provided by the Therapeutic Antibody Centre, University of Oxford (Oxford, UK). Malaria infections were initiated as in experiment one and mice were intra-muscularly injected with $75 \mu \mathrm{g}$ of either TNFR-Ig or an isotype control human purified IgG1 (Sigma) dissolved in sterile PBS (Gibco) on days 5, 6, 7 and 8 p.i. (5 mice per clone, per antibody treatment). Data on control mice from experiment two replicated our results from experiment one. TNFR-Ig treatment was started on day 5 post infection in order to minimize interference with the anti-parasitic effect of TNF- $\alpha$ early in infection (Langhorne et al. 1989; Stevenson \& Tam 1993).

\section{Monitoring parasitaemia and virulence}

Daily thin blood smears were made from tail blood and after Giemsa staining, the proportions of RBCs parasitized (parasitaemia) were counted microscopically using $\times 1000$ magnification. RBCs were counted until 20 parasites were detected in two or more separate fields of vision. This method has been shown mathematically to eliminate the bias in estimates of parasitemias which arises for statistical reasons when parasites are rare on smears (Crooks 2004). RBC densities were obtained by flow cytometery (Beckmann Coulter) from a 1:40,000 dilution of a $2 \mu 1$ sample of tail blood in Isoton 
Parasitology (2006), 133: 673-684 Cambridge University Press

doi:10.1017/S003118200600117X

solution every day p.i. in experiment one and on days 0,2 , 4, days 5-12 and then every 2 days until day 21 p.i. in experiment two, when mice were sacrificed. Mouse weights were recorded to the nearest $0.1 \mathrm{~g}$ on those days p.i. indicated above.

\section{Plasma cytokine detection.}

In experiment one, blood from individual mice was collected into heparinized tubes on the day of sacrifice (days 2,5 or 7 p.i.). In experiment two, $50 \mu$ l of tail-blood from individual mice was collected into $50 \mu 1$ heparin (Sigma) on day 7 p.i. Blood was kept on ice and centrifuged at $500 \times \mathrm{g}$ for 10 minutes $\left(4^{\circ} \mathrm{C}\right)$ to obtain plasma which was frozen at $-20^{\circ} \mathrm{C}$ until the day of assay. Plasma levels of the cytokines TNF- $\alpha$, IL-12p70, IL-6, IFN$\gamma$ and IL-10 were detected using a flow cytometric cytokine bead array $\left(\mathrm{BD}^{\mathrm{TM}} \mathrm{CBA}\right.$ Array), with slight modifications from manufacturer's instructions (BD Biosciences). Briefly, $50 \mu 1$ of plasma sample or standard were incubated in flat-bottomed 96-well plates $($ Costar®) with $25 \mu$ l of cytokine capture bead mixture (anti-cytokine-coated microspheres) in darkness, with shaking for one hour at room temperature. Wells were then washed with $200 \mu \mathrm{l}$ wash buffer $(1 \times \mathrm{PBS}$ solution $)$ and plates were spun at $200 \times \mathrm{g}$ for 5 minutes. Samples and standards were incubated with $25 \mu \mathrm{PE}$ detection reagent (phycoerythrin-conjugated anti-mouse cytokine antibodies) in darkness for one hour. Wells were then washed and beads re-suspended in $200 \mu 1$ wash buffer and analysed on FACsArray analyser (BD ${ }^{\mathrm{TM}}$ Biosciences). 
Parasitology (2006), 133: 673-684 Cambridge University Press

doi:10.1017/S003118200600117X

\section{Statistical Analysis.}

Traits fell into three categories: virulence traits (live-weight and anaemia), parasite traits (parasitaemia) and immunological traits (plasma cytokines). The minimum live-weight and $\mathrm{RBC}$ counts reached over the course of infection were used as virulence parameters for analysis of both experiments. Parasite load was quantified as the maximum asexual parasitaemia. In experiment one, parasite and virulence data from only the mice which experienced the full course of infection were analysed. Maximum plasma cytokine concentrations were analysed for all mice in that experiment. In experiment two, parasitaemia and anaemia observed after the cessation of treatment (days 9 through 16 p.i. inclusive) were also analysed and are referred to as the average post-treatment RBC count or parasitaemia. Finally, immunological data from experiment two (day 7 plasma cytokine concentrations) were analysed. Prior to statistical analysis, it was necessary to transform the data to meet the necessary normality and homogeneity-of-variances assumptions (Grafen \& Hails 2002). Box-Cox transformations were carried out on all non-normal data as an exploratory tool to help determine the optimal transformation required for normalization. In experiment one, all cytokine and $\mathrm{RBC}$ density data were $\log _{10}$ transformed prior to analysis, while in experiment two, the weight, IL-12p70 and IFN- $\gamma$ data were square root transformed, the parasitemia, IL-6 and IL-10 data were natural $\log$ transformed and the RBC parameters were inverse square root transformed. Wherever possible, data are presented in their original units for intuitive ease.

All traits mentioned above were analysed using Analysis of Variance (ANOVAs) or Covariance (ANCOVAs) in MiNITAB (release 14, MiNITAB Inc.). Explanatory variables for clone, treatment and an interaction between those terms were fitted to the 
data. Clone had up to five factor levels (CW, AS, BC, AJ and Naïve) depending on the analysis, and in experiment two, treatment had two factor levels (huIgG1 and TNFR-Ig). For all models, we first fitted the maximal model including covariate when relevant, and minimal models were obtained by removing non-significant terms $(P$-value $>0.05)$. We report $\mathrm{F}$ values and the test statistic for ANOVA as well as $P$ values, as is essential for full statistical disclosure (Olsen 2003). Naïve (uninfected) mice suffered significantly less virulence and induced significantly lower cytokine titres relative to infected mice groups regardless of clone, and so were removed from the analyses. To control for the role parasite load may play in determining virulence, a separate analysis was carried out which included maximum parasitaemia as a covariate. Finally, we tested the directional hypothesis that clone virulence increases with increasing pro-inflammatory response (TNF- $\alpha$ or IFN- $\gamma:$ IL-10) using an ordered heterogeneity test (Rice \& Gaines 1994). The $\mathrm{OH}$ test combines the $P$ value from the appropriate ANOVA $\left(P_{c}\right)$ with the Spearman's rank correlation coefficient $\left(r_{\mathrm{s}}\right)$ to calculate the test statistic $\left(r_{\mathrm{s}} P_{c}\right)$ as follows: $r_{\mathrm{s}} P_{c}=r \times(1$ - P) (Rice \& Gaines 1994).

\section{RESULTS}

Experiment one: clone-specific induction of TNF- $\alpha$.

Wild type C57BL/6 female hosts were infected with $10^{6}$ parasites of one of four genetically distinct P.c.c. clones; denoted CW, AS, BC and AJ. One mouse died during the experiment and was removed from the analyses (AJ infected mouse on day 13 p.i.). The course of these distinct primary infections, including the virulence and cytokine responses they induced, were examined. Maximum asexual parasitaemias did not differ 
significantly between clones (Fig. $1 \mathrm{~A}$; clone: $F_{3,8}=1.7, P>0.05$ ). However, clone

differences in virulence were found, with clones $\mathrm{AJ}$ and $\mathrm{BC}$ inducing lower minimum weight and RBC densities than CW or AS infections (Fig. 1B; clone: $F_{3,7}=11, P<0.01$; and Fig. 1C; clone: $F_{3,8}=6, P<0.05$, respectively). These clone differences in weight and RBC loss agree with previous studies (Mackinnon \& Read 1999a; Mackinnon \& Read 1999b; Mackinnon \& Read 2004) and were maintained even when controlling for parasite load (clone: $F_{3,6}=10, P<0.01$; and clone: $F_{3,7}=10, P<0.01$, respectively). Hence, the virulence differences observed between clones are not simply a consequence of parasite burden.

Clones differed in the cytokine profile they induced over time, with significant clone differences in day 5 IFN- $\gamma$ levels, as well as day 7 TNF- $\alpha$ and IL-6 levels (Fig. 2A; clone: $F_{3,8}=14, P<0.001$; Fig. $2 \mathrm{~B}$; clone: $F_{3,8}=5.4, P<0.05$ and Fig. $2 \mathrm{C}$; clone: $F_{3,8}=7.2, P<$ 0.05). The most virulent clones, $\mathrm{AJ}$ and $\mathrm{BC}$, induced high concentrations of proinflammatory cytokines: both induced TNF- $\alpha$; BC also induced IFN- $\gamma$, while AJ induced IL-6 (Fig. 2A, B and C). A trend was observed whereby IL-10 levels were elevated during infection with avirulent clones on day 5 p.i., but this was not significant (Fig. 2D; clone: $\left.F_{3,8}=1.2, P<0.4\right)$. Although clone differences in plasma cytokine profiles were found, only the amount of TNF- $\alpha$ induced on day 7 p.i. correlated with clone differences in virulence (Fig. $2 \mathrm{~A}$; clone: $F_{3,8}=5, P<0.05, r_{s} P_{c}=0.97, P<0.01$; and controlling for average parasitaemia; clone: $\left.F_{3,7}=6, P<0.05, r_{s} P_{c}=0.97, P<0.01\right)$. Thus, it is possible that clone differences in TNF- $\alpha$ induction around the peak of parasitaemia can help explain clone differences in virulence that are not accounted for by parasite load. 
Parasitology (2006), 133: 673-684 Cambridge University Press

doi:10.1017/S003118200600117X

Experiment two: effect of blocking TNFR1 signalling during genetically distinct malaria infections

TNFR1 signalling was blocked via the administration of a soluble TNF receptor 1 fusion protein (TNFR-Ig) during each of four distinct P.c.c. infections (as above) and in vivo virulence, parasite dynamics and host plasma cytokine response were followed throughout infection. Three mice died during the experiment and were removed from our analyses (two IgG treated, AJ infected mice and one TNFR-Ig treated, BC infected mouse on days 10 and 11 p.i.).

\section{(i) Virulence}

In vivo blocking of TNFR1 signalling protected mice against malaria-induced weight loss (Fig. 3A, B; treatment: $F_{1,28}=7, P<0.01$ ). As observed above, clone differences in weight loss were found (Fig. 3B; clone: $F_{3,28}=13, P<0.001$ ), but the level of protection conferred by treatment did not vary among clones (Fig 3B; clone $\times$ treatment: $F_{3,28}=2, P$ $=0.2$ ). For example, the protection from weight loss the TNFR-Ig treatment afforded to AS infected mice did not differ significantly from that afforded to AJ infected mice, even though the latter lost almost $2 \mathrm{~g}$ more weight. Although clone differences in posttreatment anaemia were found (Fig. $3 \mathrm{C}$ and $\mathrm{D}$; clone: $F_{3,28}=3, P<0.05$ ), in contrast to weight loss, blocking TNFR1 signalling did not affect the degree of anaemia induced (Fig. 3D; treatment and clone $\times$ treatment: $P>0.05$ in all cases). Thus, blocking TNFR1 signalling protects against malaria-induced weight loss, but the extent of this protection does not vary among clones. In addition, blocking TNFR1 signalling has no effect on anaemia induced by any of the clones. 
For the first 8 days of infection, treatment did not significantly affect asexual parasite dynamics (Fig. 4A). However, after treatment stopped on day 9 p.i., depleted mice suffered elevated parasitaemias (Fig. 4A and B; average post-treatment parasitaemia; treatment: $\left.F_{1,29}=7, P<0.05\right)$. We analysed several aspects of the parasite data presented in Fig. 4A. While there were clone differences in average parasitaemia and day of peak parasitemia (Fig. 4A; clone: $F_{3,29}=5, P<0.01$; and clone: $F_{3,29}=10, P<0.001$, respectively) and near significant differences in both the maximum and average posttreatment parasitaemia (clone: $F_{3,29}=2, P=0.09$; and clone: $F_{3,29}=3, P=0.06$, respectively), the magnitude of these clone differences were unaffected by treatment (clone $\times$ treatment: $P>0.05$ in all cases). Thus, blocking TNFR1 signalling had similarly negligible effects on parasite kinetics for all the clones.

\section{(iii) Per-parasite virulence}

To differentiate between the roles parasite load and immunopathology play in driving malaria virulence (Graham et al. 2005) we examined the effects of clone and treatment on virulence by statistically taking maximum parasitaemia into account. Although parasite load was a significant predictor of weight loss $\left(F_{1,27}=8, P<0.01\right)$, it did not affect clone differences in weight loss, nor the protective effect of treatment on minimum weight (clone: $F_{3,27}=10, P<0.001$, treatment: $F_{1,27}=7, P<0.05$, clone $\times$ treatment: $F_{3,27}=2 P$ $=0.1$ ). These results show that TNFR-1 signalling contributes towards malaria-induced weight loss, independently of parasite load or parasite genotype. Thus, the virulence 
differences among clones and treatments depicted in Fig. 3B and D are not due to parasitaemia differences alone.

For a given parasite burden, TNFR-Ig treated mice suffered greater anaemia from day 9 p.i. onwards after treatment ended (treatment: $F_{1,27}=5, P<0.05$ ). In contrast, clone differences in post-treatment anaemia were removed once parasite load was taken into account (clone: $F_{3,27}=2, P=0.2$ and clone $\times$ treatment: $F_{3,27}=0.4, P=0.8$ ). Parasite load alone thus explains clone differences in post-treatment anaemia.

\section{(iv) Cytokines associated with immunopathology}

To examine the induction of cytokines that might contribute to the clone or treatment effects on virulence, we measured the levels of several plasma cytokines on day 7 p.i. during experiment two. Clones differed significantly in the induction of plasma IFN- $\gamma$, IL-6 and IL-10 on day 7 p.i. (Table 1). Treatment, on the other hand, only significantly affected plasma IL-6, with TNFR-1 blockade causing elevated IL-6 levels on day 7 p.i., regardless of P.c.c. clone (Table 1).

We are interested in whether clone virulence or avirulence relates to the development of pro- or anti-inflammatory cytokine responses. In this regard, we found that controlling for parasite load, day 7 plasma IFN- $\gamma$ levels helped to explain both the minimum live-weight and anaemia reached $\left(F_{1,26}=21, P<0.001\right.$ and $F_{1,26}=8, P<0.01$, respectively). Thus, just as we investigated the role parasite load plays in driving virulence in the previous section, we now observe that our virulence results (Fig. 3B, D) relate to IFN- $\gamma$ levels. Thus, elevated IFN- $\gamma$ around the peak of infection may have detrimental virulence outcomes (Kremsner et al. 1992; Waki et al. 1992). High ratios of 
pro- to anti-inflammatory cytokines have been associated with malaria virulence in the field (Dodoo et al. 2002). In agreement with this, our data show that the ratio of IFN- $\gamma$ to IL-10 is higher for more virulent P.c.c. infections, regardless of parasite load (Fig. 5; clone: $F_{3,28}=3.2, P<0.05$; and clone: $\left.r_{s} P_{c}=0.966, P<0.01\right)$. Thus, it is possible that clone differences in IFN- $\gamma$ induction, or in the regulation of IFN- $\gamma$ by anti-inflammatory cytokines, contribute towards clone differences in malaria virulence.

\section{DISCUSSION}

Malaria virulence may be induced directly by the parasite or via immunemediated mechanisms, which include TNF- $\alpha$ (Clark \& Chaudhri 1988a; Clark \& Chaudhri 1988b; Grau et al. 1987; Kern et al. 1992). For example, high levels of serum TNF- $\alpha$ correlate with poor outcome and disease severity in both rodent (Clark \& Chaudhri 1988a; Clark \& Chaudhri 1988b) and human (Kwiatkowski 1990; Kwiatkowski et al. 1990) malaria infections. We hypothesized that P.c.c. clones may achieve high virulence by (i) inducing an exacerbated TNF- $\alpha$ response, (ii) growing to high parasite densities, or (iii) via a combination of these factors.

In experiment one, we observed that more virulent malaria clones induced higher day 7 plasma TNF- $\alpha$ levels (Fig. 2A), even when parasite load was taken into account. In experiment two, TNFR1 signalling was blocked during genetically distinct infections, with the expectation that clone virulence schedules would be differentially affected and possibly eliminated. However, we found that regardless of parasite genotype, blocking TNFR-1 signalling ameliorated weight loss (Fig. 3A and B), and had little effect on anaemia or parasitaemia (Fig. 3C, 3D and 4A, respectively). These data suggest that the 
Parasitology (2006), 133: 673-684 Cambridge University Press

doi:10.1017/S003118200600117X

contribution of TNF- $\alpha$ to malaria virulence does not depend on P.c.c. genotype. Thus, the previously reported effects of TNF- $\alpha$ on both the virulence and parasitaemia during P.c.c. AS infections (Jacobs et al. 1996; Li \& Langhorne 2000; Sam et al. 1999) appear representative of those observed across the suite of P.c.c. clones we studied.

Separating the pathogenic effects of an overzealous immune response from those of parasite burden is extremely difficult (Graham et al. 2005). By adding parasitaemia to statistical models, we were able to show that clone differences in the induction of plasma TNF- $\alpha$ on day 7 p.i. are not completely driven by parasite load and correlate with virulence. In the same respect, lethal and non-lethal $P$. yoelii infections have been shown to differ in their ability to induce early TGF- $\beta$, despite no significant differences in parasite load at that time (Omer et al. 2003). Furthermore, the enhanced disease severity of IL-10 deficient mice infected with the parasites Trypanosoma cruzi (Hunter et al. 1997), Toxoplasma gondii (Gazzinelli et al. 1996), Helicobacter hepaticus (Kullberg et al. 1998) or P. chabaudi (Li et al. 1999) did not correlate with increased parasite burdens, but rather with an increased inflammatory response. These data suggest that for many parasite species, disease severity is at least partly immune-mediated and independent of parasite load.

There are several possible mechanisms by which different malaria genotypes might induce TNF- $\alpha$, independent of parasite burden. For example, it is plausible that the cellular source of TNF- $\alpha$, or alternatively the trigger for TNF- $\alpha$ release, may differ during infections with genetically distinct parasites. It has been shown that during P.c.c AS infections, macrophages (Stevenson et al. 1992) and dendritic cells (Seixas et al. 2001) can produce TNF- $\alpha$ upon direct stimulation by the parasite in vitro, and CD4+ T 
Parasitology (2006), 133: 673-684 Cambridge University Press

doi:10.1017/S003118200600117X

cells have also been shown to produce TNF- $\alpha$ during malaria infection (Hirunpetcharat et al. 1999). If P.c.c. clones differ in ability to induce these cellular responses, this could explain the differential amplification of TNF- $\alpha$ we observe in the plasma. It would be of interest to determine what the cellular source(s) of cytokines are during infection with our distinct clones, and also to examine whether clones differ in antigenicity. A substantial amount is currently known regarding P.c.c. AS immunology (Langhorne et al. 2004; Stevenson \& Riley 2004), but all other P.c.c. clones await similar attention.

Our data suggest that TNFR1 signalling is dispensable (between days 5-8 p.i.) for the ultimate control of malaria infection, regardless of parasite genotype. To our knowledge, the reagents used in this study do not distinguish between TNF- $\alpha$ and the closely related cytokine Lymphotoxin- $\alpha$ (LT- $\alpha)$, which can both signal through TNFR1 and thus we have been cautious to attribute treatment effects to TNFR1 signalling rather than TNF- $\alpha$ itself. In any case, cytokine redundancies may help explain the relatively small effects of TNFR-Ig treatment. For example, IL-6 or IFN- $\gamma$ could be compensating for the absence of TNFR1 signalling. It has been proposed that differences in induction of key anti-inflammatory cytokines, which act to down-regulate the nascent proinflammatory response, may also contribute to malaria pathology (Artavanis-Tsakonas et al. 2003; Dodoo et al. 2002). In this regard we found ratios of plasma IFN- $\gamma$ to IL-10 correlate with clone virulence (Fig. 5). Future experiments designed to block other key cytokines (including IL-6 and IFN- $\gamma$ ), possibly in combination, during diverse malaria infections may reveal cytokines that differentially affect clone virulence. Finally, it is possible that TNFR-independent pathways for virulence determination exist, for example both the induction of nitric oxide $(\mathrm{NO})$, or generation of reactive oxygen species have 
been implicated in the pathogenesis of severe malaria (Anstey et al. 1996; Clark et al. 1983; Griffiths et al. 2001), and may contribute to clone virulence differences observed.

In conclusion, our results demonstrate that blocking TNFR1 signalling protects against weight loss during P.c.c infections, regardless of parasite genotype or parasitaemia. However, a role for parasite genotype in determining the contribution of IFN- $\gamma$ and IL-6-mediated pathology to virulence is not ruled out by this study. Hence, studies aimed at investigating the contribution of cytokine-mediated pathways to the virulence induced by genetically diverse malaria infections deserve further attention. Clearer definition of the root causes of malarial virulence, including the proportion due to immunopathology, would help to identify essential targets which could be used in the treatment of malaria.

\section{ACKNOWLEDGEMENTS}

G. H. Long is supported by a Wellcome Trust studentship and A. L. Graham is supported by the Leverhulme Trust and the School of Biological Sciences, University of Edinburgh.

We thank the staff of the March House, University of Edinburgh, for excellent animal husbandry, Dr. P. Bird (Therapeutic Antibody Centre, University of Oxford, UK) for providing TNFR-Ig, S. MacCall for technical assistance in performing CBA assays and H. Ferguson for helpful comments on the manuscript. This work was supported by the Wellcome Trust (069299/Z/02/A). 


\section{REFERENCES}

Alles H K, M. K. N., Carter R. 1998 Malaria Mortality Rates in South Asia and in Africa: Implications for Malaria Control. Parasitology Today 14, 369-375.

Anstey, N. M., Weinberg, J. B., Hassanali, M. Y., Mwaikambo, E. D., Manyenga, D., Misukonis, M. A., Arnelle, D. R., Hollis, D., McDonald, M. I. \& Granger, D. L. 1996 Nitric oxide in Tanzanian children with malaria: inverse relationship between malaria severity and nitric oxide production/nitric oxide synthase type 2 expression. J Exp Med 184, 557-67.

Artavanis-Tsakonas, K., Tongren, J. E. \& Riley, E. M. 2003 The war between the malaria parasite and the immune system: immunity, immunoregulation and immunopathology. Clin Exp Immunol 133, 145-52.

Ashkenazi, A., Marsters, S. A., Capon, D. J., Chamow, S. M., Figari, I. S., Pennica, D., Goeddel, D. V., Palladino, M. A. \& Smith, D. H. 1991 Protection against endotoxic shock by a tumor necrosis factor receptor immunoadhesin. Proc Natl Acad Sci U S A 88, 10535-9.

Bate, C. A. \& Kwiatkowski, D. 1994 A monoclonal antibody that recognizes phosphatidylinositol inhibits induction of tumor necrosis factor alpha by different strains of Plasmodium falciparum. Infect Immun 62, 5261-6.

Bate, C. A., Taverne, J., Bootsma, H. J., Mason, R. C., Skalko, N., Gregoriadis, G. \& Playfair, J. H. 1992 Antibodies against phosphatidylinositol and inositol monophosphate specifically inhibit tumour necrosis factor induction by malaria exoantigens. Immunology 76, 35-41. 
Bate, C. A., Taverne, J. \& Playfair, J. H. 1988 Malarial parasites induce TNF production by macrophages. Immunology 64, 227-31.

Bate, C. A., Taverne, J. \& Playfair, J. H. 1989 Soluble malarial antigens are toxic and induce the production of tumour necrosis factor in vivo. Immunology 66, 600-5.

Chotivanich, K., Udomsangpetch, R., Simpson, J. A., Newton, P., Pukrittayakamee, S., Looareesuwan, S. \& White, N. J. 2000 Parasite multiplication potential and the severity of Falciparum malaria. J Infect Dis 181, 1206-9.

Clark, I. A., Alleva, L. M., Mills, A. C. \& Cowden, W. B. 2004 Pathogenesis of malaria and clinically similar conditions. Clin Microbiol Rev 17, 509-39.

Clark, I. A. \& Chaudhri, G. 1988a The balance of useful and harmful effects of TNF, with special reference to malaria. Ann Inst Pasteur Immunol 139, 305-6.

Clark, I. A. \& Chaudhri, G. 1988b Tumour necrosis factor may contribute to the anaemia of malaria by causing dyserythropoiesis and erythrophagocytosis. $\mathrm{Br} J$ Haematol 70, 99-103.

Clark, I. A., Cowden, W. B., Butcher, G. A. \& Hunt, N. H. 1983 Free oxygen radicals in malaria. Lancet 1, 359-60.

Crooks, L. 2004 Gametocyte Investment in Malaria. PhD thesis University of Edinburgh.

de Roode, J. C., Pansini, R., Cheesman, S. J., Helinski, M. E., Huijben, S., Wargo, A. R., Bell, A. S., Chan, B. H., Walliker, D. \& Read, A. F. 2005 Virulence and competitive ability in genetically diverse malaria infections. Proc Natl Acad Sci U $S$ A 102, 7624-8. 
Dodoo, D., Omer, F. M., Todd, J., Akanmori, B. D., Koram, K. A. \& Riley, E. M. 2002 Absolute levels and ratios of proinflammatory and anti-inflammatory cytokine production in vitro predict clinical immunity to Plasmodium falciparum malaria. J Infect Dis 185, 971-9.

Ferguson, H. M., Mackinnon, M. J., Chan, B. H. \& Read, A. F. 2003 Mosquito mortality and the evolution of malaria virulence. Evolution Int J Org Evolution 57, 2792-804.

Gazzinelli, R. T., Wysocka, M., Hieny, S., Scharton-Kersten, T., Cheever, A., Kuhn, R., Muller, W., Trinchieri, G. \& Sher, A. 1996 In the absence of endogenous IL-10, mice acutely infected with Toxoplasma gondii succumb to a lethal immune response dependent on CD4+ T cells and accompanied by overproduction of IL12, IFN-gamma and TNF-alpha. J Immunol 157, 798-805.

Grafen, A. \& Hails, R. 2002 Modern Statistics for the Life Sciences Oxford University Press, Oxford.

Graham, A. L., Lamb, T. J., Read, A. F. \& Allen, J. E. 2005 Malaria-filaria coinfection in mice makes malarial disease more severe unless filarial infection achieves patency. $J$ Infect Dis 191, 410-21.

Grau, G. E., Del Giudice, G. \& Lambert, P. H. 1987 Host immune response and pathological expression in malaria: possible implications for malaria vaccines. Parasitology 94 Suppl, S123-37.

Grau, G. E., Taylor, T. E., Molyneux, M. E., Wirima, J. J., Vassalli, P., Hommel, M. \& Lambert, P. H. 1989 Tumor necrosis factor and disease severity in children with falciparum malaria. $N$ Engl J Med 320, 1586-91. 
Gravenor, M. B., McLean, A. R. \& Kwiatkowski, D. 1995 The regulation of malaria parasitaemia: parameter estimates for a population model. Parasitology $110(\mathbf{P t}$ 2), 115-22.

Griffiths, M. J., Ndungu, F., Baird, K. L., Muller, D. P., Marsh, K. \& Newton, C. R. 2001 Oxidative stress and erythrocyte damage in Kenyan children with severe Plasmodium falciparum malaria. Br J Haematol 113, 486-91.

Hirunpetcharat, C., Finkelman, F., Clark, I. A. \& Good, M. F. 1999 Malaria parasitespecific Th1-like T cells simultaneously reduce parasitemia and promote disease. Parasite Immunol 21, 319-29.

\section{Hunter, C. A., Ellis-Neyes, L. A., Slifer, T., Kanaly, S., Grunig, G., Fort, M.,} Rennick, D. \& Araujo, F. G. 1997 IL-10 is required to prevent immune hyperactivity during infection with Trypanosoma cruzi. J Immunol 158, 3311-6.

Jacobs, P., Radzioch, D. \& Stevenson, M. M. 1996 A Th1-associated increase in tumor necrosis factor alpha expression in the spleen correlates with resistance to bloodstage malaria in mice. Infect Immun 64, 535-41.

Jacobs, R. L. 1964 Role of P-Aminobenzoic Acid in Plasmodium Berghei Infection in the Mouse. Exp Parasitol 15, 213-25.

Kebaier, C., Louzir, H., Chenik, M., Ben Salah, A. \& Dellagi, K. 2001 Heterogeneity of wild Leishmania major isolates in experimental murine pathogenicity and specific immune response. Infect Immun 69, 4906-15.

\section{Kern, P., Hemmer, C. J., Gallati, H., Neifer, S., Kremsner, P., Dietrich, M. \&} Porzsolt, F. 1992 Soluble tumor necrosis factor receptors correlate with parasitemia and disease severity in human malaria. J Infect Dis 166, 930-4. 
Parasitology (2006), 133: 673-684 Cambridge University Press

doi:10.1017/S003118200600117X

Kremsner, P. G., Neifer, S., Chaves, M. F., Rudolph, R. \& Bienzle, U. 1992

Interferon-gamma induced lethality in the late phase of Plasmodium vinckei

malaria despite effective parasite clearance by chloroquine. Eur J Immunol 22, 2873-8.

Kullberg, M. C., Ward, J. M., Gorelick, P. L., Caspar, P., Hieny, S., Cheever, A., Jankovic, D. \& Sher, A. 1998 Helicobacter hepaticus triggers colitis in specificpathogen-free interleukin-10 (IL-10)-deficient mice through an IL-12- and gamma interferon-dependent mechanism. Infect Immun 66, 5157-66.

Kwiatkowski, D. 1990 Tumour necrosis factor, fever and fatality in falciparum malaria. Immunol Lett 25, 213-6.

Kwiatkowski, D., Hill, A. V., Sambou, I., Twumasi, P., Castracane, J., Manogue, K. R., Cerami, A., Brewster, D. R. \& Greenwood, B. M. 1990 TNF concentration in fatal cerebral, non-fatal cerebral, and uncomplicated Plasmodium falciparum malaria. Lancet 336, 1201-4.

Langhorne, J., Albano, F. R., Hensmann, M., Sanni, L., Cadman, E., Voisine, C. \& Sponaas, A. M. 2004 Dendritic cells, pro-inflammatory responses, and antigen presentation in a rodent malaria infection. Immunol Rev 201, 35-47.

Langhorne, J., Cross, C., Seixas, E., Li, C. \& von der Weid, T. 1998 A role for B cells in the development of $\mathrm{T}$ cell helper function in a malaria infection in mice. Proc Natl Acad Sci U S A 95, 1730-4.

Langhorne, J., Meding, S. J., Eichmann, K. \& Gillard, S. S. 1989 The response of CD4+ T cells to Plasmodium chabaudi chabaudi. Immunol Rev 112, 71-94. 
Li, C., Corraliza, I. \& Langhorne, J. 1999 A defect in interleukin-10 leads to enhanced malarial disease in Plasmodium chabaudi chabaudi infection in mice. Infect Immun 67, 4435-42.

Li, C. \& Langhorne, J. 2000 Tumor necrosis factor alpha p55 receptor is important for development of memory responses to blood-stage malaria infection. Infect Immun 68, 5724-30.

Li, C., Sanni, L. A., Omer, F., Riley, E. \& Langhorne, J. 2003 Pathology of Plasmodium chabaudi chabaudi infection and mortality in interleukin-10-deficient mice are ameliorated by anti-tumor necrosis factor alpha and exacerbated by antitransforming growth factor beta antibodies. Infect Immun 71, 4850-6.

Li, C., Seixas, E. \& Langhorne, J. 2001 Rodent malarias: the mouse as a model for understanding immune responses and pathology induced by the erythrocytic stages of the parasite. Med Microbiol Immunol (Berl) 189, 115-26.

Mackinnon, M. J., Gaffney, D. J. \& Read, A. F. 2002 Virulence in rodent malaria: host genotype by parasite genotype interactions. Infect Genet Evol 1, 287-96.

Mackinnon, M. J. \& Read, A. F. 1999a Genetic relationships between parasite virulence and transmission in the rodent malaria Plasmodium chabaudi. Evolution Int J Org Evolution 53, 689-703.

Mackinnon, M. J. \& Read, A. F. 1999b Selection for high and low virulence in the malaria parasite Plasmodium chabaudi. Proc Biol Sci 266, 741-8.

Mackinnon, M. J. \& Read, A. F. 2004 Virulence in malaria: an evolutionary viewpoint. Philos Trans R Soc Lond B Biol Sci 359, 965-86. 
Molyneux, M. E., Engelmann, H., Taylor, T. E., Wirima, J. J., Aderka, D., Wallach, D. \& Grau, G. E. 1993 Circulating plasma receptors for tumour necrosis factor in Malawian children with severe falciparum malaria. Cytokine 5, 604-9.

Molyneux, M. E., Taylor, T. E., Wirima, J. J. \& Grau, G. E. 1991 Tumour necrosis factor, interleukin-6, and malaria. Lancet 337, 1098.

Mordue, D. G., Monroy, F., La Regina, M., Dinarello, C. A. \& Sibley, L. D. 2001 Acute toxoplasmosis leads to lethal overproduction of Th1 cytokines. J Immunol $167,4574-84$.

Naessens, J., Kitani, H., Nakamura, Y., Yagi, Y., Sekikawa, K. \& Iraqi, F. 2005 TNF-alpha mediates the development of anaemia in a murine Trypanosoma brucei rhodesiense infection, but not the anaemia associated with a murine Trypanosoma congolense infection. Clin Exp Immunol 139, 405-10.

Olsen, C. H. 2003 Review of the use of statistics in infection and immunity. Infect Immun 71, 6689-92.

Omer, F. M., de Souza, J. B. \& Riley, E. M. 2003 Differential induction of TGF-beta regulates proinflammatory cytokine production and determines the outcome of lethal and nonlethal Plasmodium yoelii infections. J Immunol 171, 5430-6.

Rice, W. R. \& Gaines, S. D. 1994 Extending nondirectional heterogeneity tests to evaluate simply ordered alternative hypotheses. Proc Natl Acad Sci U S A 91, 225-6.

Ritter, U., Mattner, J., Rocha, J. S., Bogdan, C. \& Korner, H. 2004 The control of Leishmania (Leishmania) major by TNF in vivo is dependent on the parasite strain. Microbes Infect 6, 559-65. 
Sam, H. \& Stevenson, M. M. 1999 Early IL-12 p70, but not p40, production by splenic macrophages correlates with host resistance to blood-stage Plasmodium chabaudi AS malaria. Clin Exp Immunol 117, 343-9.

Sam, H., Su, Z. \& Stevenson, M. M. 1999 Deficiency in tumor necrosis factor alpha activity does not impair early protective Th1 responses against blood-stage malaria. Infect Immun 67, 2660-4.

Seixas, E., Cross, C., Quin, S. \& Langhorne, J. 2001 Direct activation of dendritic cells by the malaria parasite, Plasmodium chabaudi chabaudi. Eur J Immunol 31, 29708.

Snow, R. W., Guerra, C. A., Noor, A. M., Myint, H. Y. \& Hay, S. I. 2005 The global distribution of clinical episodes of Plasmodium falciparum malaria. Nature 434, 214-7.

Stevenson, M. M., Huang, D. Y., Podoba, J. E. \& Nowotarski, M. E. 1992 Macrophage activation during Plasmodium chabaudi AS infection in resistant C57BL/6 and susceptible A/J mice. Infect Immun 60, 1193-201.

Stevenson, M. M. \& Riley, E. M. 2004 Innate immunity to malaria. Nat Rev Immunol 4, $169-80$.

Stevenson, M. M. \& Tam, M. F. 1993 Differential induction of helper T cell subsets during blood-stage Plasmodium chabaudi AS infection in resistant and susceptible mice. Clin Exp Immunol 92, 77-83.

Stevenson, M. M., Tam, M. F., Wolf, S. F. \& Sher, A. 1995 IL-12-induced protection against blood-stage Plasmodium chabaudi AS requires IFN-gamma and TNF- 
Parasitology (2006), 133: 673-684 Cambridge University Press

doi:10.1017/S003118200600117X

alpha and occurs via a nitric oxide-dependent mechanism. J Immunol 155, 254556.

Su, Z. \& Stevenson, M. M. 2002 IL-12 is required for antibody-mediated protective immunity against blood-stage Plasmodium chabaudi AS malaria infection in mice. J Immunol 168, 1348-55.

Torre, D., Speranza, F. \& Martegani, R. 2002 Role of proinflammatory and antiinflammatory cytokines in the immune response to Plasmodium falciparum malaria. Lancet Infect Dis 2, 719-20.

Waki, S., Uehara, S., Kanbe, K., Ono, K., Suzuki, M. \& Nariuchi, H. 1992 The role of $\mathrm{T}$ cells in pathogenesis and protective immunity to murine malaria. Immunology 75, 646-51.

Walliker, D., Carter, R. \& Morgan, S. 1971 Genetic recombination in malaria parasites. Nature 232, 561-2. 
TABLE 1. Effects of TNFR1 blockade and P. chabaudi clone on levels of plasma IL-6, IFN- $\gamma$, IL-10 and IL-12p70 on day 7 post infection

$$
\text { Plasma cytokine concentration }(\mathrm{pg} / \mathrm{ml})^{\mathrm{a}}
$$

Clone

Treatment

IL-6 ${ }^{\mathrm{c}}$

IFN- $\gamma$

IL-10

IL-12p70

CW

huIgG1

$47.5 \pm 1$

$5.3 \pm 0.5$

$12.9 \pm 1.9$

$31.7 \pm 1.5$

TNFR-Ig

$151.4 \pm 9.2$

$7.6 \pm 0.3$

$14.4 \pm 1.0$

$32.8 \pm 1.3$

AS

huIgG1

$70.1 \pm 8.4$

$46.3 \pm 4.8$

$33.0 \pm 5.3$

$29.0 \pm 3.6$

TNFR-Ig

$155.9 \pm 19.3$

$43.2 \pm 0.9$

$55.4 \pm 7.7$

$56.5 \pm 2.9$

BC

huIgG1

111.2 \pm 9.1

$57.1 \pm 3.1$

$0^{\mathrm{d}}$

TNFR-Ig

$90.6 \pm 8.2$

$28.8 \pm 1.4$

$15.2 \pm 2.4$

$20.5 \pm 2.3$

$39.7 \pm 2.6$

AJ

huIgG1

TNFR-Ig

$20.9 \pm 1.7$

$13.8 \pm 1.2$

$16.6 \pm 2.6$

$34.0 \pm 3.7$

$55.2 \pm 3.2$

$25.6 \pm 1.1$

$25.7 \pm 2.7$

$33.4 \pm 1.0$

significance

of clone

$F_{3,29}=3$
$P<0.05$

$F_{3,29}=5$

$F_{3,29}=3$

$P<0.01$

$P<0.05$

ns

$P<0.05$

${ }^{a}$ Mice were infected with $10^{6} \mathrm{pRBCs}$ of one of four P.c.c clones (CW, AS, BC or AJ), blood was obtained from individual mice on day 7 p.i., and plasma levels of IL-6, IFN- $\gamma$, IL-10 and IL-12 p70 were determined. Data are means of five mice $( \pm S E)$, per clone per treatment, except where deaths occurred as noted in the Results section.

${ }^{\mathrm{b}}$ Effects of clone on levels of plasma cytokines was investigated via ANOVA and significance tests are indicated.

c TNFR-Ig treated mice had significantly higher IL-6 levels compared to control huIgG1 mice $\left(F_{1,29}=5 P<0.05\right)$, regardless of clone.

${ }^{\mathrm{d}} 0$, values below detection limits of assay. 
Fig.1. Effect of Plasmodium chabaudi parasite clone on the kinetics of asexual parasitaemia (A), minimum weight (B) and minimum RBC density (C) reached during genetically distinct single-clone infections. Infections were initiated with $10^{6}$ RBCs parasitised with one of P.c.c. clone CW, AS, BC or AJ. Parasitaemia and virulence measurements were collected every day. Mice were sequentially sacrificed on days 2, 5, 7 and 14 p.i. Each line represents the mean of 12, 9, 6 and 3 mice up to these time points, respectively (+/- SE) (A) and each bar represents the least square mean of 3 mice (+/- SE) (B and C).

Fig. 2. Effect of Plasmodium chabaudi parasite clone (CW, AS, BC or AJ) on the levels of plasma TNF- $\alpha$ (A), IFN- $\gamma$ (B), IL-6 (C) and IL-10 (D). Single-clone P.c.c. infections were initiated and blood was obtained from individual mice on day of sacrifice (days 2, 5 and 7 p.i.). Plasma cytokine levels were determined using a flow cytometric cytokine bead array (see Materials and Methods). Each bar represents the mean of 3 mice (+/- SE).

Fig. 3. Effect of parasite clone and TNFR-Ig treatment on the kinetics of malaria-induced weight and red blood cell loss. Daily or every other day, mice were weighed and red blood cell counts obtained. Changes in live-weight over time (A), minimum live-weight (B), kinetics of RBC density over time (C), and minimum RBC density (D) during the course of primary infection with one of four P.c.c. clones (CW, AS, BC or AJ) are shown for TNFR-Ig treated mice (open symbols and hatched bars) or control IgG-treated mice (solid symbols and solid bars). Each line or bar represents the mean of 5 mice (+/- SE), except where deaths occurred as noted in the results section. 
Fig. 4. Effect of parasite clone and TNFR-Ig treatment on the kinetics of asexual parasitaemia. Plots represent the percentage parasitised RBCs during infection with one of four P.c.c. clones (CW, AS, BC or AJ) in TNFR-Ig treated mice (open symbols) or control IgG-treated mice (solid symbols) (A). Bars represent the least square mean of post-treatment asexual parasitaemia (day 9-16 inclusive), broken down by clone and treatment (B). Each line or bar represents the mean of 5 mice $(+/-\mathrm{SE})$, except where deaths occurred as noted in the results section.

Fig. 5. Effect of parasite clone on the ratio of plasma IFN- $\gamma$ to IL-10 on day 7 postinfection, for each of four P.c.c clones (CW, AS, BC or AJ). Blood was obtained from individual mice on day 7 p.i. and plasma levels of IFN- $\gamma$ and IL-10 were determined. The natural $\log$ transformation was applied to data to meet the necessary homogeneity-ofvariance and normality assumptions. Bars are least square mean of 5 mice per infecting clone $( \pm$ SE), except where deaths occurred as noted in Results section. 


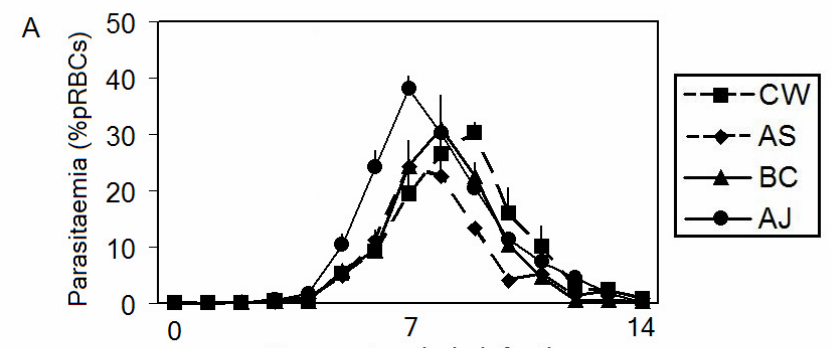

Day post malaria infection
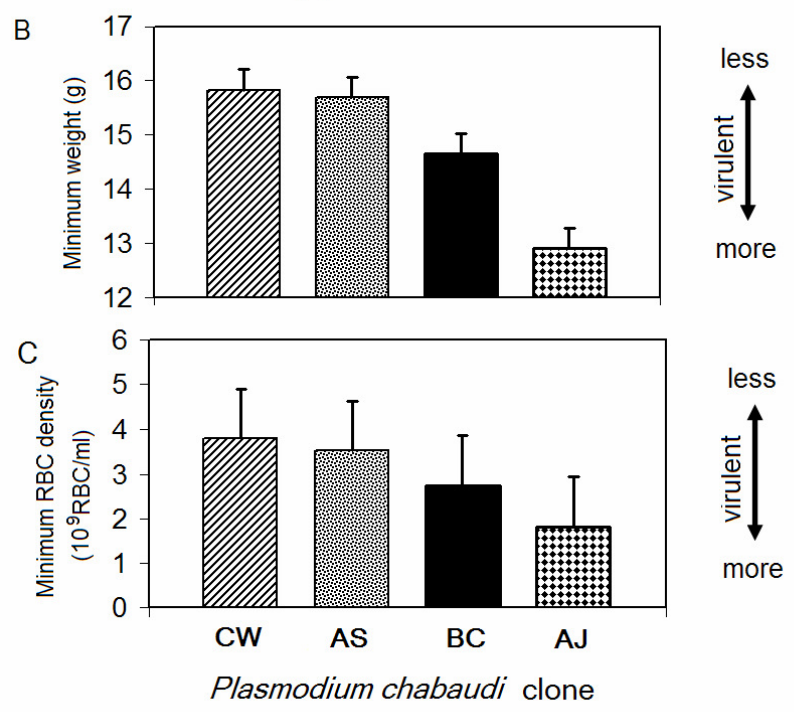

less
more

Plasmodium chabaudi clone

Fig. 1A, B \& C.

TNF- $\alpha$ and rodent malaria genotype (G. H. Long et al) 

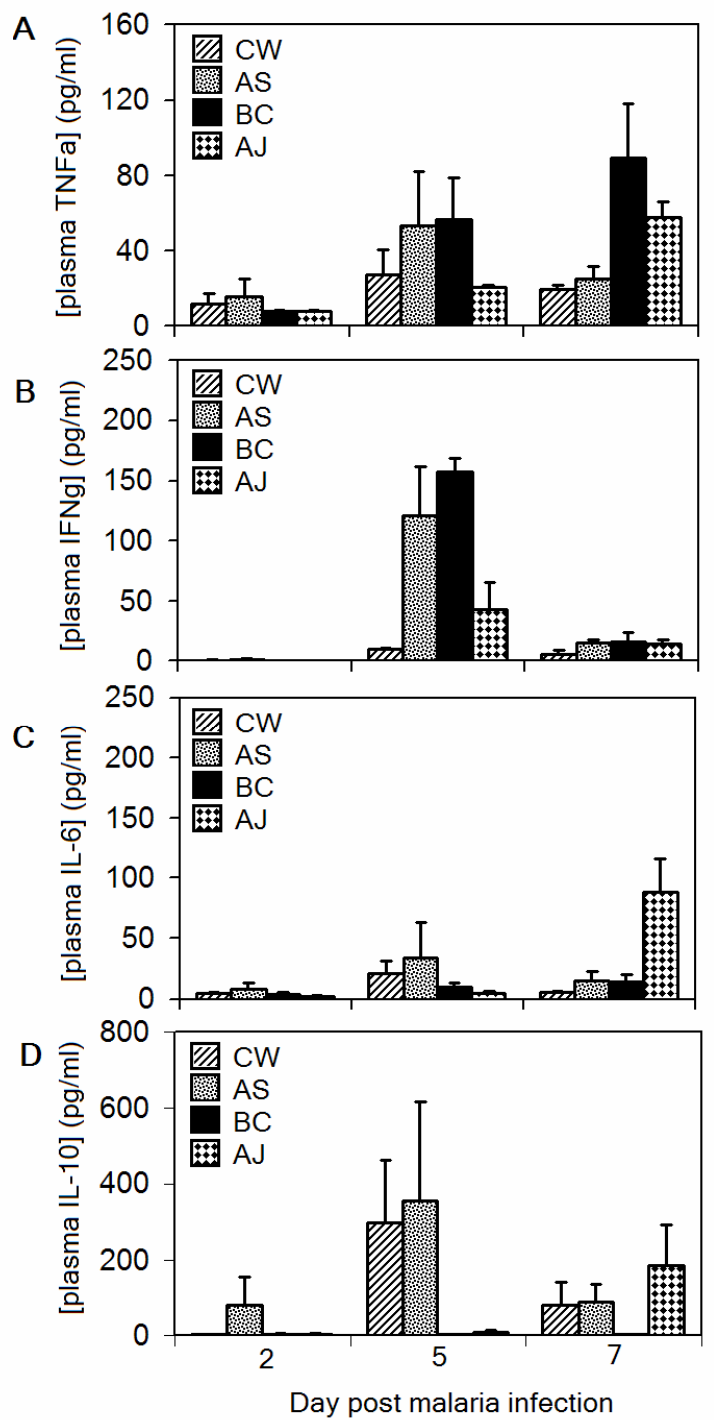

Fig. 2A, B, C \& D.

TNF- $\alpha$ and rodent malaria genotype (G. H. Long et al) 

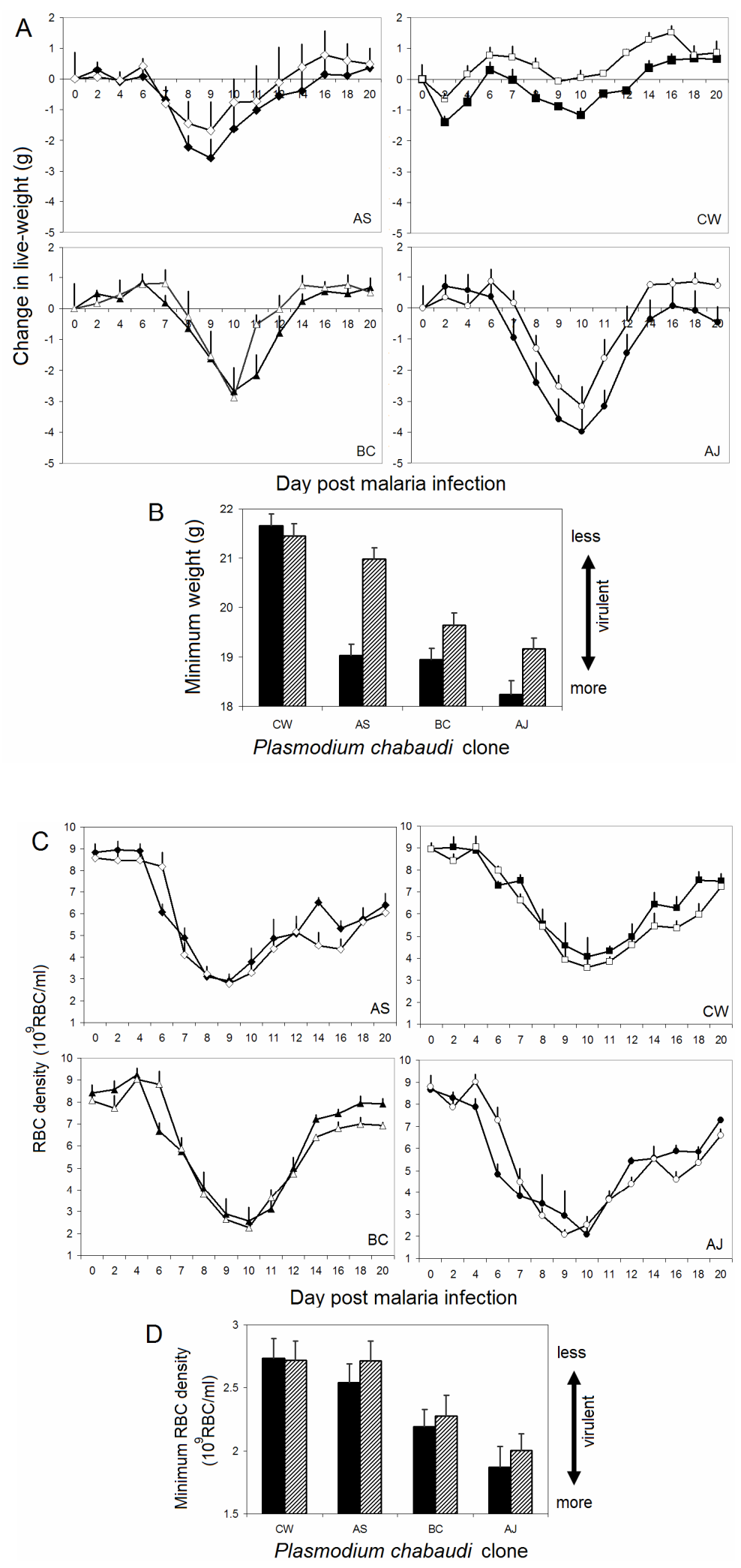

Fig. 3 A, B, C \& D.

TNF- $\alpha$ and rodent malaria genotype (G. H. Long et al) 


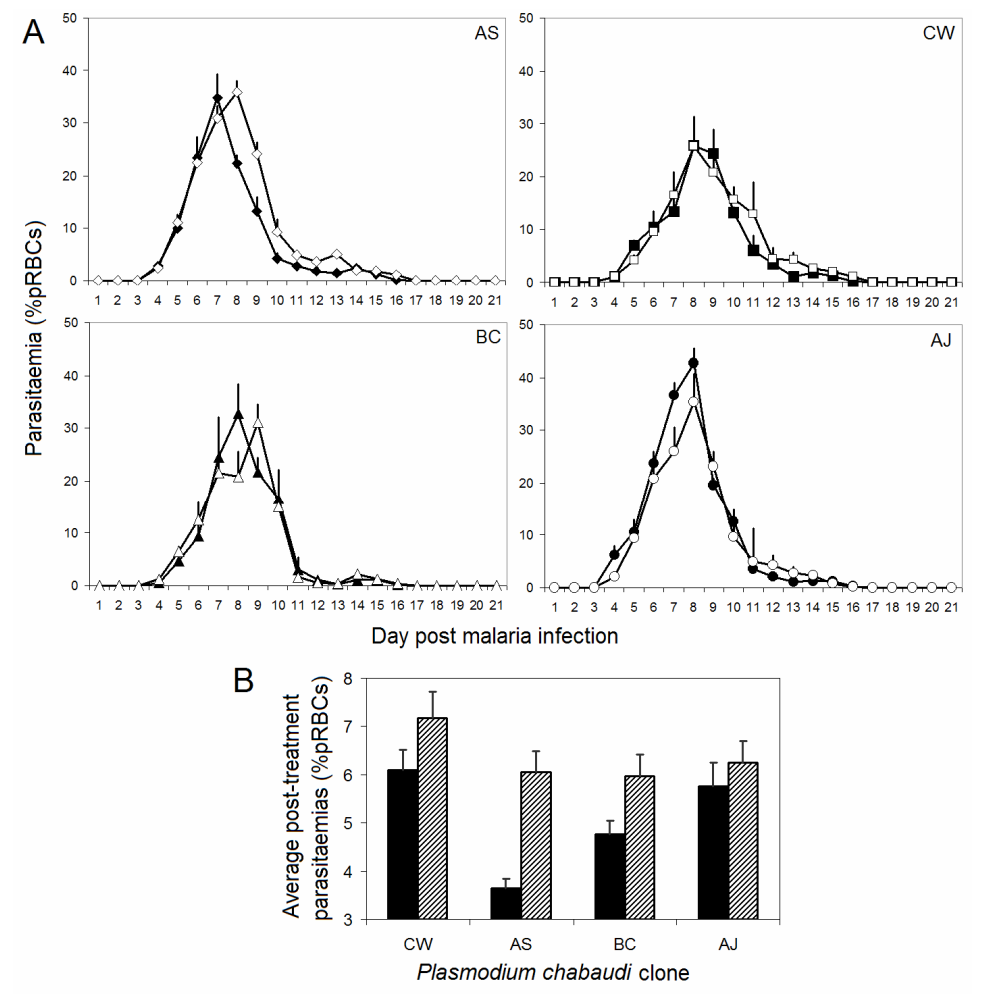

Fig. 4 A \& B.

TNF- $\alpha$ and rodent malaria genotype (G. H. Long et al) 


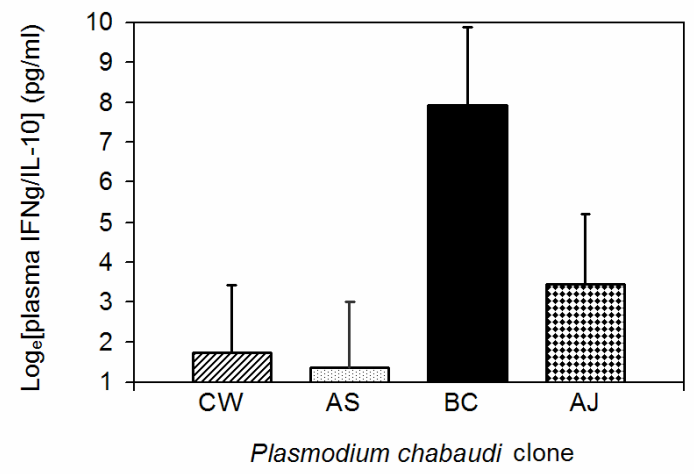

Fig. 5.

TNF- $\alpha$ and rodent malaria genotype (G. H. Long et al) 